\title{
Building up mass in the centers of late type galaxies $\dagger$
}

\author{
E. Schinnerer ${ }^{1}$, T. Böker ${ }^{2}$, E. Emsellem ${ }^{3}$, U. Lisenfeld ${ }^{4,5}$ and \\ D. Downes ${ }^{6}$
}

${ }^{1}$ Max Planck Institute for Astronomy, Königstuhl 17, D-69117 Heidelberg, Germany

${ }^{2}$ European Space Agency, Dept. RSSD, Keplerlaan 1, 2200 AG Noordwijk, Netherlands

${ }^{3}$ Université de Lyon 1, Observatoire de Lyon, CNRS, UMR 5574, Centre de Recherche

Astrophysique de Lyon, Ecole Normale Supérieure de Lyon, France

${ }^{4}$ Dept. Física Teórica y del Cosmos, Facultad de Ciencias, Universidad de Granada, 18071 Granada, Spain

${ }^{5}$ Instituto de Astrofísica de Andalucía, CSIC, Apdo. 3004, 18080 Granada, Spain

${ }^{6}$ Institut de RadioAstronomie Millimétrique, 300 rue de la Piscine, Domaine Universitaire, 38406 Saint Martin d'Hères, France

\begin{abstract}
We present highest angular resolution $\left(\sim 1^{\prime \prime}\right.$ and $\left.0.35^{\prime \prime}\right)$ mm-interferometric observations of the $\mathrm{HCN}(1-0),{ }^{12} \mathrm{CO}(1-0)$ and ${ }^{12} \mathrm{CO}(2-1)$ line emission in the central $300 p c$ of the late-type spiral galaxy NGC 6946. The data, obtained with the IRAM Plateau de Bure Interferometer $(\mathrm{PdBI})$ shows for the first time a molecular gas spiral in the inner $\sim 10^{\prime \prime}(270 \mathrm{pc})$ with a large concentration of molecular gas $\left(M_{H_{2}} \sim 1.6 \times 10^{7} M_{\odot}\right)$ within the inner $60 p c$, The gas distribution in the central $50 \mathrm{pc}$ has been resolved and is consistent with a gas ring or spiral driven by a bar. Both the distribution of the molecular gas as well as its kinematics can be well explained by the influence of an inner stellar bar of about $400 p c$ length as tested via a qualitative model for the gas flow. NGC 6946 is a prime example of molecular gas kinematics being driven by a small-scale, secondary stellar bar.

For the first time, it is possible to directly compare the location of (dense) giant molecular clouds with that of (optically) visible HII regions in space-based images. We use the $3 \mathrm{~mm}$ continuum and the HCN emission to estimate in the central $50 \mathrm{pc}$ the star formation rates in young clusters that are still embedded in their parent clouds and hence are missed in optical and near-IR surveys of star formation. The amount of embedded star formation is about 1.6 times as high as that measured from HII regions alone, and appears roughly evenly split between ongoing dust-obscured star formation and very young giant molecular cloud cores that are just beginning to form stars. The build-up of central mass seems to have continued over the past $\geqslant 10$ Myrs, to have occurred in an extended (albeit small) volume around the nucleus, and to be closely related to the presence of an inner bar.
\end{abstract}

Keywords. galaxies: ISM, galaxies: kinematics and dynamics, galaxies: nuclei, galaxies: individual (NGC 6946)

\section{Introduction}

About $75 \%$ of spiral galaxies have a compact ( $\sim$ few pc diameter), photometrically distinct nuclear star cluster. The young age of most nuclear clusters indicates that these galaxies must recently have had significant inflow (of up to $10^{8} \mathrm{M}_{\odot}$ ) of molecular gas into their very nuclei in order to trigger the cluster formation. While there is consensus that

$\dagger$ Based on observations carried out with the IRAM Plateau de Bure Interferometer. IRAM is supported by INSU/CNRS (France), MPG (Germany) and IGN (Spain). 

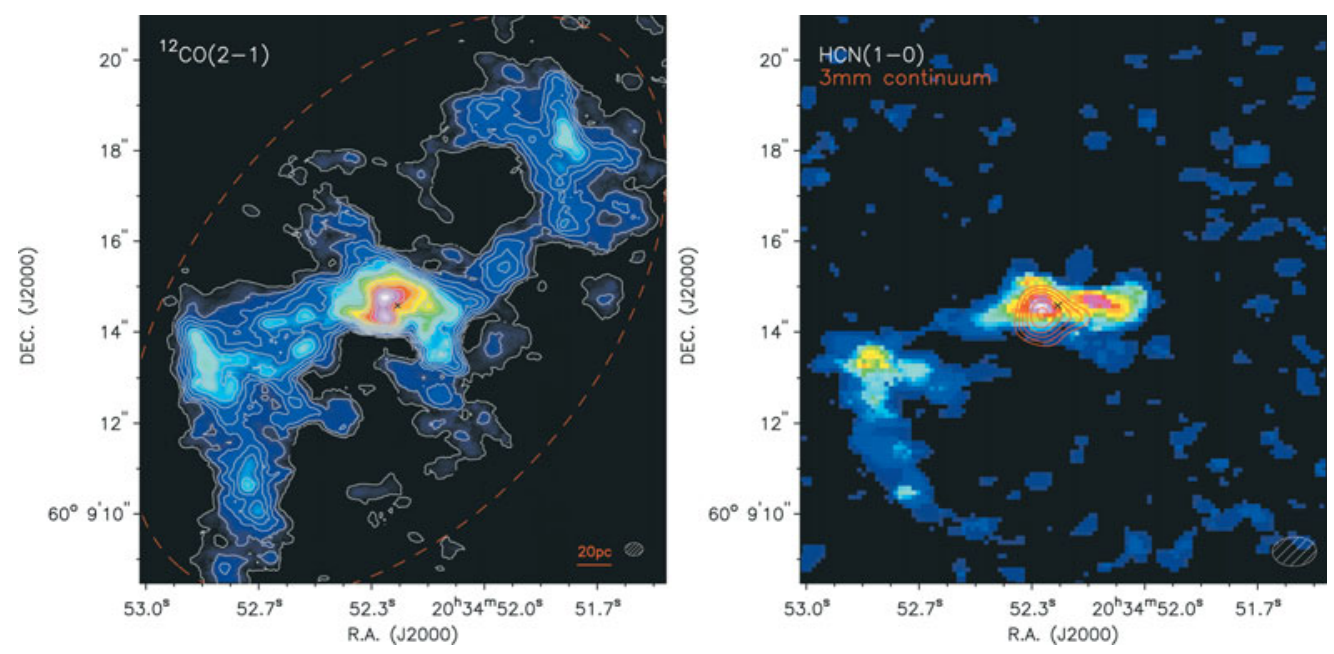

Figure 1. Left: PdBI intensity map of the $\mathrm{CO}(2-1)$ line emission at $0.4 " \times 0.3$ " resolution (color and contours) in the central $300 \mathrm{pc}$ of the late-type spiral galaxy NGC 6946 (Schinnerer et al. 2007). The molecular gas forms a spiral structure very reminiscent of the gas/dust geometry predicted by dynamical models of barred galaxies. The contours start at $0.6 \mathrm{Jy}$ beam ${ }^{-1} \mathrm{~km} \mathrm{~s}^{-1}$ with the same step size. The red dashed ellipse indicates the location and size of the inner bar (Schinnerer et al. 2006). Right: Comparison of the $\operatorname{HCN}(1-0)$ intensity map (color) and the $3 \mathrm{~mm}$ continuum (contours) at $1.0 " \times 0.7$ " resolution. Showing that there is abundant dense molecular gas to form stars and that indeed recent star formation within the inner $50 \mathrm{pc}$ of the center has been building up stellar mass over the past $\geqslant 10$ Myrs (Schinnerer et al. 2007). The contours start at $3 \sigma$ and are in steps of $1 \sigma=0.21 \mathrm{mJy}$ beam $^{-1}$. The position of the dynamical center (black cross), beam size and linear scale are shown in the bottom right corners.

large-scale stellar bars are the main mechanism to transport molecular gas to the inner kiloparsec there is no clear picture on how to bridge the last few 100 parsecs.

NGC 6946 is an almost well-studied face-on late-type barred spiral galaxy with a prominent nuclear starburst. The starburst history of the inner $8 "(\approx 230 \mathrm{pc})$ can be described by two recent events (about 5 Myrs and 15 Myrs ago) that each converted about $(5-10) \times 10^{7} M_{\odot}$ of molecular gas mass into stars (Engelbracht et al. 1996). These authors infer a high extinction of $A_{V} \approx 10$ towards the nuclear region which is surprising given the almost face-on geometry. In addition to the large-scale bar responsible for NGC 6946 being classified as SB, near-infrared observations by Elmegreen et al. 1998 found a secondary (nested) "mini-bar" of $\sim 270 p c$ length. NGC 6946 is a prime target for studying the process of central mass build-up: it is nearby, $(D \approx 5.5 M p c$, i.e. $27 p c /$ ", has abundant nuclear star formation, and it has a very bright, fairly well-resolved molecular gas structure which Schinnerer et al. 2006, Schinnerer et al. 2007 recently studied in detail by ${ }^{12} \mathrm{CO}$ line emission observed with the PdBI.

\section{Observations}

NGC 6946 was observed with the IRAM Plateau de Bure interferometer (PdBI) between 2002 and 2006 in the ${ }^{12} \mathrm{CO}(1-0),{ }^{12} \mathrm{CO}(2-1)$, and $\mathrm{HCN}(1-0)$ line. The simultaneous observations at $3 \mathrm{~mm}$ and $1 \mathrm{~mm}$ covered the two ${ }^{12} \mathrm{CO}$ lines in the old $\mathrm{AB}$ configuration of $\mathrm{PdBI}$, while the $\mathrm{CO}(2-1)$ line and $\mathrm{HCN}(1-0)$ line were observed with the new extended A configuration with baselines of up to $760 \mathrm{~m}$. The data reduction was done in a standard way using the IRAM software package of GILDAS. For the CLEANing we defined a CLEAN box based on the 0th moment maps of the respective lines (for details see 
Schinnerer et al. 2006, Schinnerer et al. 2007). The final resolution of the data are $~ 1 "$ for the $3 \mathrm{~mm}$ lines and $\sim 0.35$ " for the $\mathrm{CO}(2-1)$ line at $1 \mathrm{~mm}$. The achieved rms per 6 $\mathrm{km} / \mathrm{s}$ wide channel is about 2 to $5 \mathrm{mJy} /$ beam for the $3 \mathrm{~mm}$ and $1 \mathrm{~mm}$ lines.

\section{Results}

Our new ${ }^{12} \mathrm{CO}(2-1)$ data resolve the molecular gas into a spiral-like distribution (Fig. 1) with three high-luminosity clumps located at the dynamical center and on either end of the spiral. Strong streaming motions are associated with the straight gas lanes of this spiral structure and are even observed within the inner few 10 parsec. This geometry is very reminiscent of the gas lanes expected to form due to a stellar bar potential. The observed CO kinematics can be best explained by a nuclear stellar bar which funnels molecular gas into the central $50 \mathrm{pc}$. Our detailed modeling (Fig. 2) shows that the deviations from circular velocity can be attributed to such a stellar bar which has also been detected in NIR images of NGC 6946 (Elmegreen et al. 1998). Our analysis suggests that inner bars are indeed capable of funneling gas towards galactic nuclei to within at least a few tens of parsec

The combined analysis of the amount of dense gas present (via the $\mathrm{HCN}(1-0)$ line), freefree radio continuum from young star forming regions (3 $\mathrm{mm}$ continuum) and archival HST Pa $\alpha$ emission from HII regions shows that they all have a slightly different spatial distribution highlighting that they all trace different evolutionary stages of star formation. We find about twice as much embedded star formation (traced by HCN and $3 \mathrm{~mm}$ continuum) as star formation which has already emerged from its dust cocoon (traced by $\operatorname{Pa} \alpha$ ). This suggests that stellar mass has been building up with a typical star formation rate of $0.1 \mathrm{M}_{\odot} \mathrm{yr}^{-1}$ over the past $\geqslant 10$ Myrs.

\section{Summary}

Our detailed analysis of the molecular gas dynamics in the central $300 p c$ of NGC 6946, as traced by the ${ }^{12} \mathrm{CO}$ lines can be summarized as follows (Schinnerer et al. 2006, Schinnerer et al. 2007): The molecular gas is distributed in an S-shaped spiral structure which can be explained if the gas is responding to an inner stellar bar resulting in very well ordered gas kinematics. The molecular gas in the central 2" (60 pc) is resolved into a 'twin-peak' geometry when seen at 0.35 " resolution. The central CO morphology can plausibly be explained by the gas being distributed in a ring at the position of the inner Lindblad resonance. It hosts a starburst which, to a large extent, is still embedded and thus invisible at optical/NIR wavelengths. The starburst that has been ongoing for several Myrs suggests that the gas kinematics of the nuclear bar are the dominating factor and stellar feedback might at most be important locally.

\section{References}

Elmegreen, D. M., Chromey, F. R., \& Santos, M. 1998, A.J., 116, 1221

Engelbracht, C. W., Rieke, M. J., Rieke, G. H., \& Latter, W. B. 1996, Ap.J., 467, 227

Schinnerer, E., Böker, T., Emsellem, E., \& Lisenfeld, U. 2006, Ap.J. 649, 181

Schinnerer, E., Böker, T., Emsellem, E., \& Downes, D. 2007, A\&̈A Letters 462, L27 

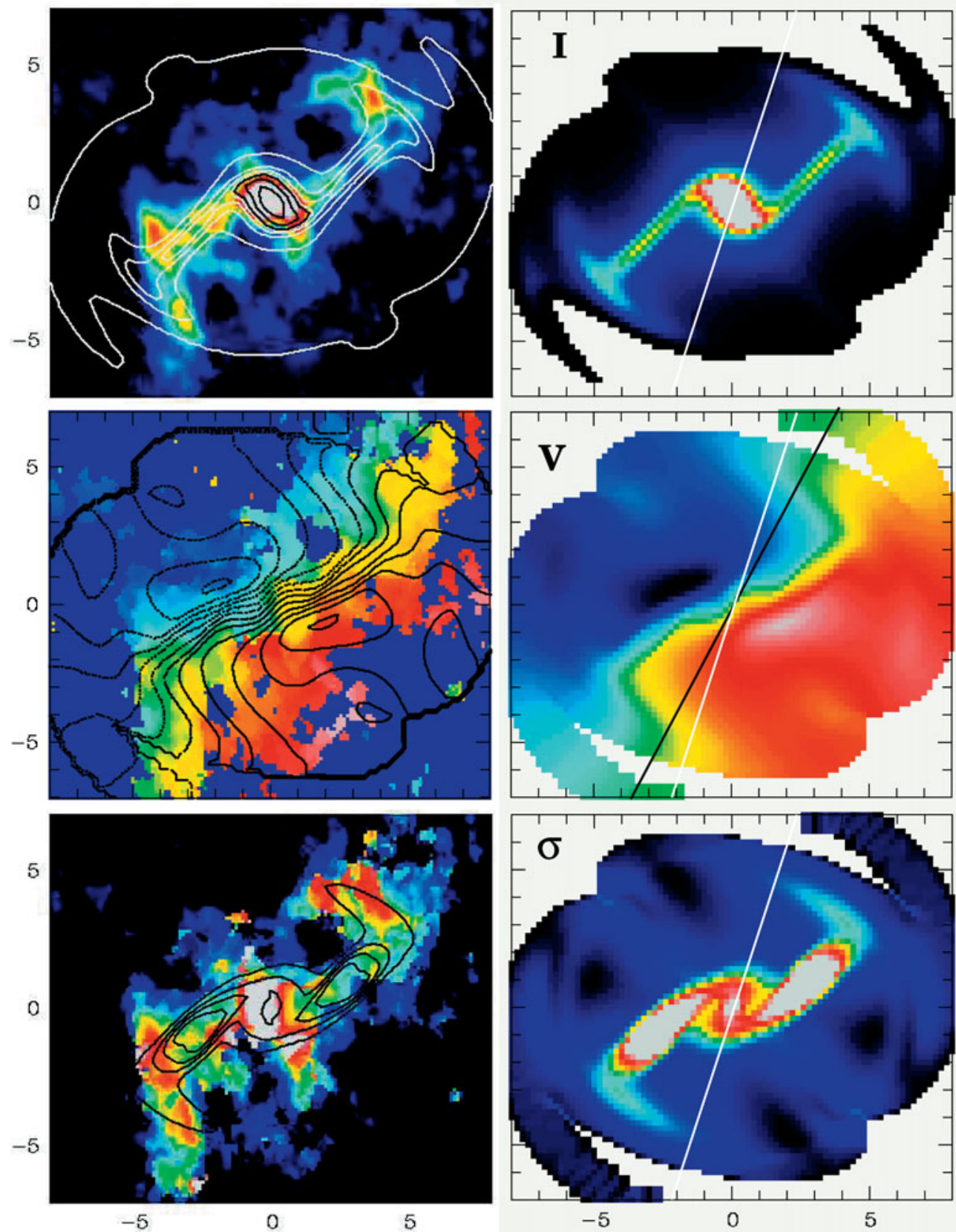

Figure 2. The molecular gas distribution and kinematics are very well explained by the influence of a nuclear stellar bar. Comparison between the intensity maps (top), the velocity fields (middle) and the velocity dispersion maps (bottom) of the gas component of the barred model (right: color, left: contours) and the observed $\mathrm{CO}(2-1)$ line emission (left: color). In the right panels, we indicate the apparent position angle of the bar in the model (white dashed line) and the line-of-nodes of the unperturbed axisymmetric potential (black dotted line). 